\title{
The electrocardiogram signal morphology analysis based on convolutional neural network
}

\author{
M Zavoyskih $^{1}$, A Korobeynikov ${ }^{1,2}$, A Menlitdinov ${ }^{2}$, V Lyuminarskiy $^{1}$ and \\ Yu Kuzelin ${ }^{3}$ \\ ${ }^{1}$ Kalashnikov Izhevsk State Technical University, Studencheskaya 7, Izhevsk, Russia, 426069 \\ ${ }^{2}$ IzhTeleMed Ltd., Lenina, 110, Izhevsk, Russia, 426009 \\ ${ }^{3}$ Republican Clinical Diagnostic Center, Lenina 87B, Izhevsk, Russia, 426009 \\ e-mail: grizz8575@gmail.com,kav33@inbox.ru
}

\begin{abstract}
The analysis of electrocardiogram signal morphology based on convolutional neural network is considered. Input data is obtained by splitting the signal into cardiac cycles. The calculation the average cycle is performed to exclude the artefacts. The Haar wavelet transform of the average cycle is performed. The images of size 200x6 are input data for the recognition system: 200 - number of counts constituting the cycle; 6 - number of Haar transform time scales. This work is a reconsideration of the previous work of the authors. The training samples base of marked cardiac cycle segments is the same (1500 cycles): the average cycle and the segment's start and end indexes. In the previous work, the original composite system consisting of several modules was used as a recognition system. In current work it is proposed to use the convolutional neural network with the special structure: 4 convolutional layers, 2 dense layers, and 200 outputs for every of 3 segment. The recognition system based on neural network showed results slightly superior to the previous system. The percent of acceptable localization of the segments is the following: $\mathrm{P}-82.2 \%$, QRS $-88.7 \%$, and $\mathrm{T}-85.4 \%$. The proposed system effectively solves the problem using the standard modules of modern artificial neural networks.
\end{abstract}

\section{Introduction}

Among the many methods of research, observation and monitoring of human condition, the leading place belongs to electrocardiography (ECG). The need to monitor the ECG during anesthesia, resuscitation, intensive care and diagnosis is beyond all doubt. The ECG signal carries a large amount of information, and a detailed automatic analysis of the patient's ECG signal allows to timely generate alarms that prevent death, as well as to make prognostic conclusions.

When processing the ECG signal, taking the advantages of modern computing resources, it is required to automatically analyze the shape of the ECG signal cycle (cardiocycle), i.e. automatically recognize the characteristic segments and determine their time and amplitude parameters. [1] 
An example of the ECG signal cardiac cycle shape and it's characteristic points is shown in Fig. 1. It is common in cardiology to analyze the segments, i.e. the regions between the characteristic points: the segment of $\mathrm{P}$ (points $\mathrm{P}_{0} \ldots \mathrm{P}_{1}$ ), QRS segment of a point (Q...S), and the segment $\mathrm{T}$ (point $\mathrm{T}_{0} \ldots \mathrm{T}_{1}$ ). The morphology analysis input receives information obtained at the stage of splitting the ECG signal into cycles: an array of cardiocycle signals accumulated over a some period of time. [2-3]

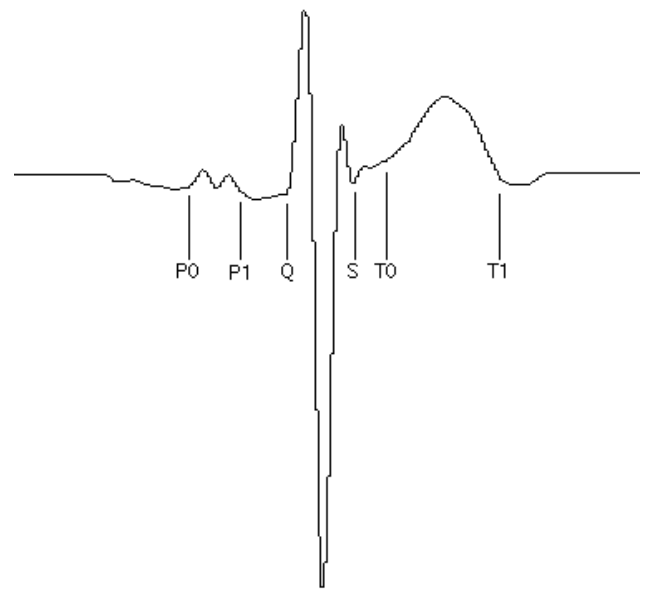

Figure 1. The average shape of the cardiac cycle.

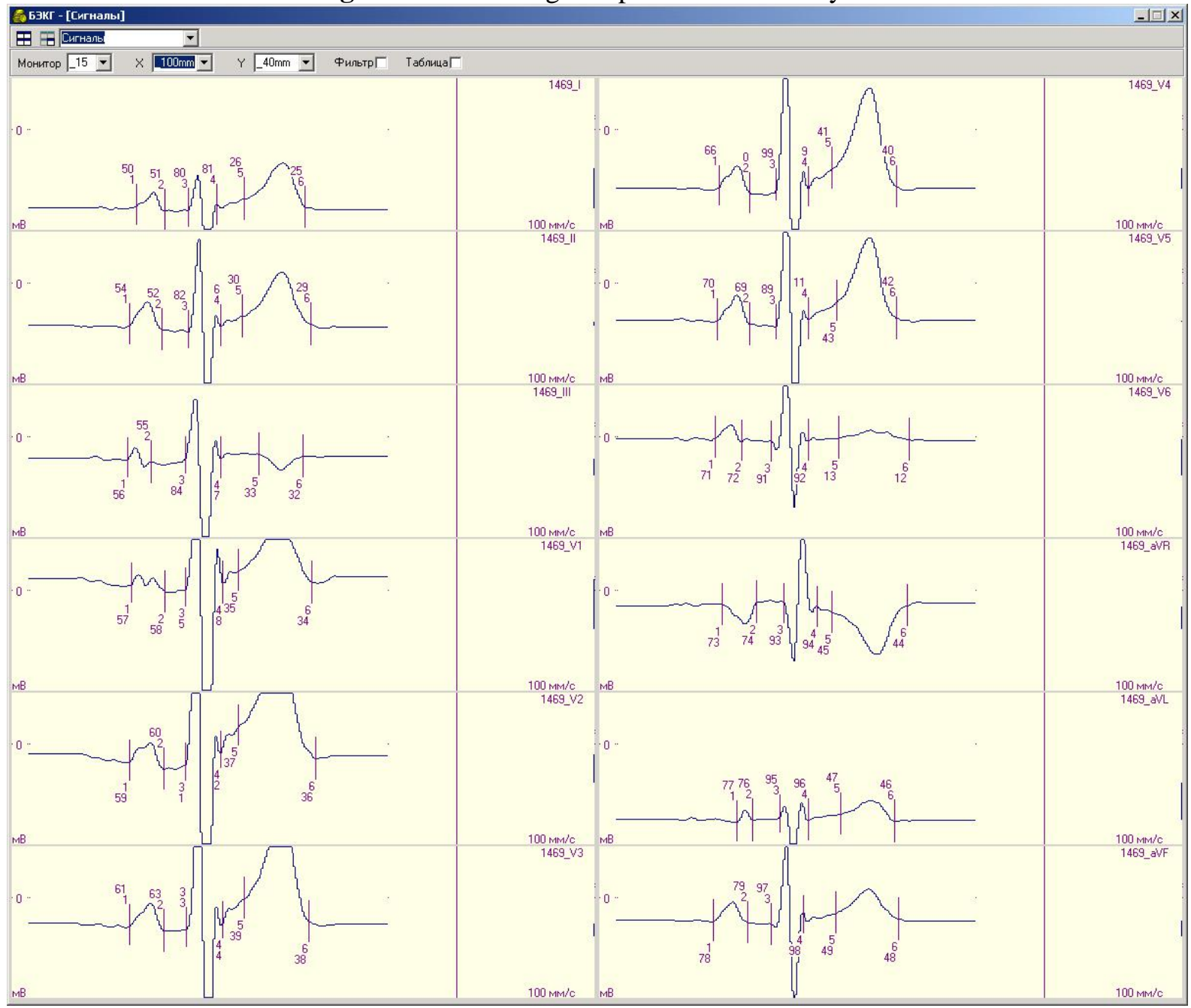

Figure 2. The software used to obtain the training sample base of marked cardiac cycles segments. 


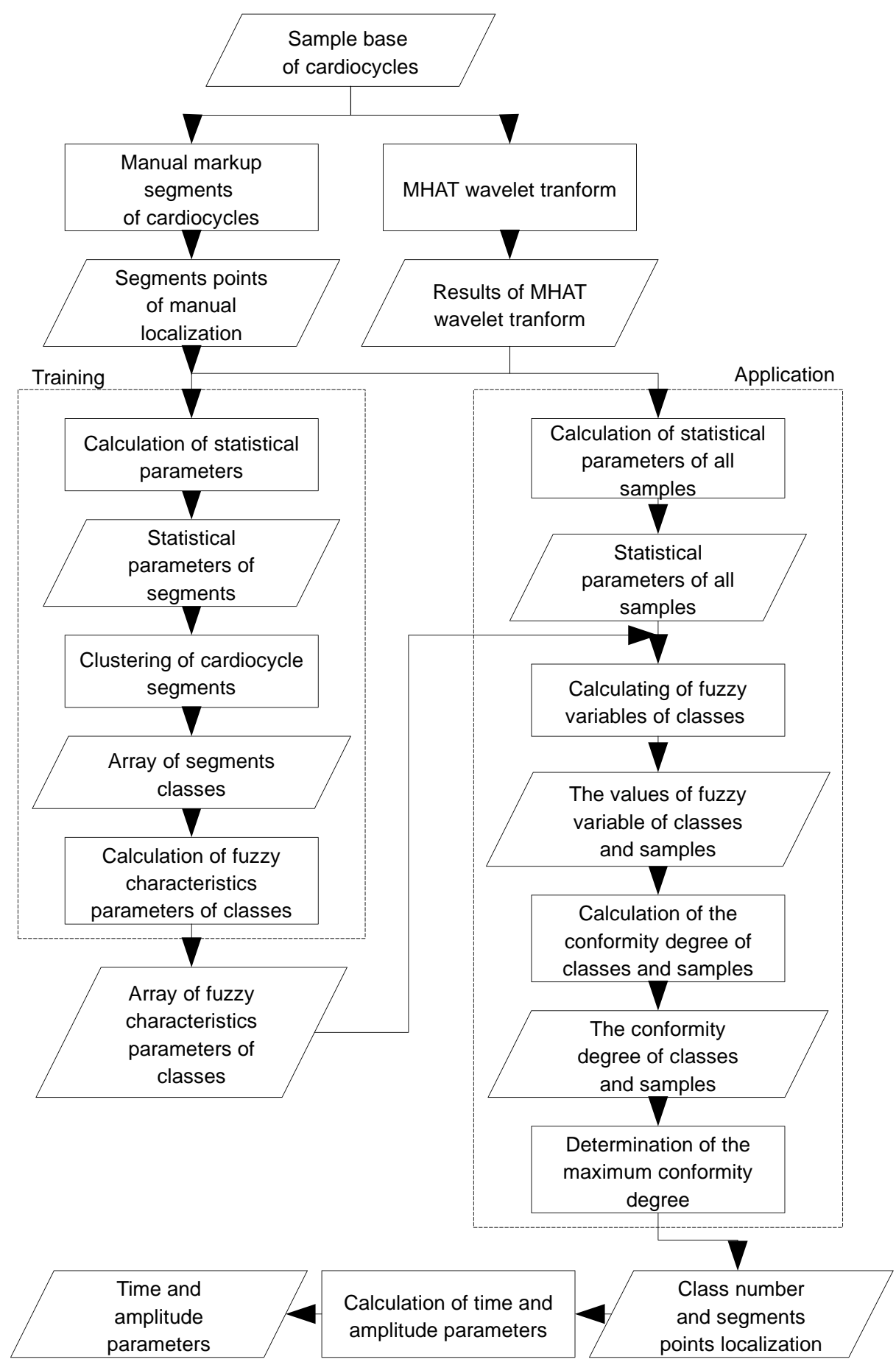

Figure 3. The stages of the previous version of the cardiac cycle morphology analysis.

\section{The previous version of the cardiac cycle morphology analysis}

Based on the fact that the normal cardiocycle shape changes slowly over time and each current cardiocycle can be distorted by artifacts and noises, it makes sense to recognize the characteristic segments of average cardiocycle shape. The average cardiocycle of several (5-20) cardiocycles having a high correlation coefficient with each other is calculated. Then the selected cardiocycles are averaged pointwise. An example of the obtained signal is shown in Fig. 1 [2]. In the following stages, the shape (characteristic points and segments) of the cardiocycle is recognized. 
The training of the recognition system is performed on the basis of the information provided after the manual cardiocycle marking, i.e. the localization of the start and end indexes of the segments $P$, QRS, and T is known. Such information can be formed using specially developed software. Marking of cardiocycles segments should be made by a medical specialist in this area. The screenshot example of the software developed by LLC Izhtelemed for performing of such marking is presented on Fig. 2. [4]

In [2] it was proposed to use a special algorithm based on the pattern recognition of the ECG signal (Fig. 3.). An original composite system consisting of several modules was developed.

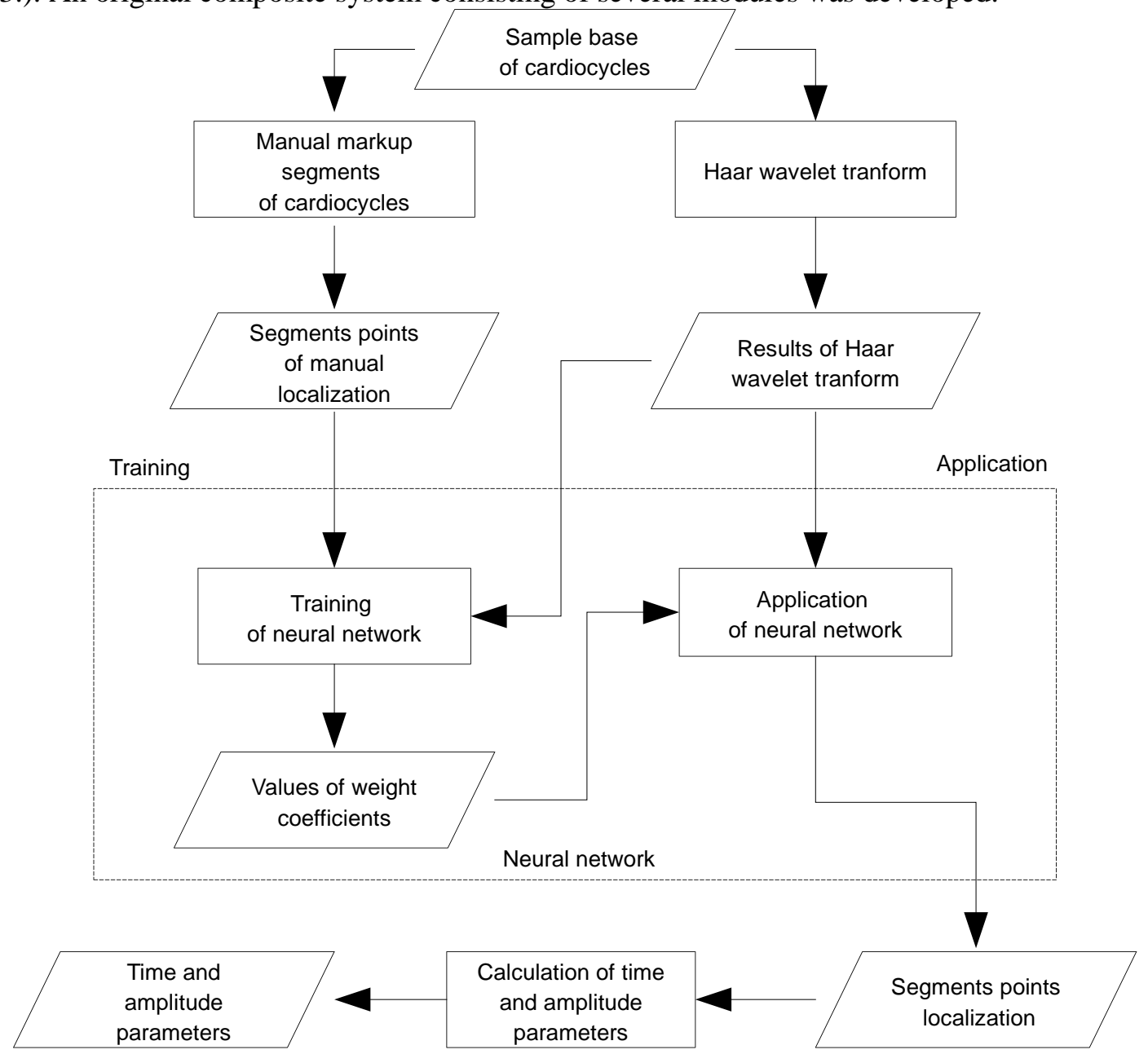

Figure 4. The stages of the cardiac cycle morphology analysis based on neural network.

\section{The cardiac cycle morphology analysis based on neural network}

In this paper, a reconsideration of the approach based on the pattern recognition of the cardiocycle morphology is carried out, and it is proposed to use an artificial neural network (ANN) as a recognition system of cardiocycle segments. Fig. 4 presents the stages of morphology analysis based on convolutional ANN.

Currently, most studies on ECG analysis algorithms use wavelet transform [5-7]. In the course of the study in [2] it was found that the wavelet transform application of the signal simplifies the ECG cardiac cycle segmentation. In this paper, in contrast to the previous work [2], the Haar wavelet transform of the average cardiocycle was chosen due to the simplicity of its implementation [8]:

$$
d^{k+1_{i}}=\frac{x^{k_{2 i}-x^{k_{2 i+1}}}}{2}, x^{k+1_{i}}=\frac{x^{k_{2 i}+x^{k_{2 i+1}}}}{2},
$$

where $\mathrm{x}$ is the signal for which the wavelet transform of the next time scale is calculated; $\mathrm{d}$ is the result of the wavelet transform calculation; $\mathrm{k}$ is the time scale number; $\mathrm{i}$ is the signal point index. 
As a result of wavelet transform of each cardiocycle at different time scales, a matrix of halfdifferences is obtained based on which the image is formed. This image then will be fed to the ANN input layer. A total of 6 time scales of transformation is used, an example is shown on Fig. 7.

At the moment, most researchers in signal, speech and image recognition algorithms use a neural network [9-13]. Some researchers combine wavelets and neural network [14-15].

To solve the problem of cardiocycle segments localization based on the wavelet transform results, the apparatus of convolutional ANN (which have been successfully used for image processing in recent years) was chosen.

The first convolutional ANN (which borrowed for computer science the ideas embodied by nature in the visual cortex) was the ANN of Kunihiko Fukushima (Neocognitron, 1975-1980) [16]. The convolutional network in already quite modern form appeared in the works of Yann LeCun (LeNet, 1989). [9]

The input data for the ANN in this paper are the Haar wavelet transform results performed on the average cardiocycle, presented in the form of images of size 200x6, where 200 - the number of counts constituting the cardiocycle ( 1 second), 6 - the number of time scales of the Haar wavelet transform.

The base of the cardiac cycle segments recognition system are the modules of the convolutional ANN. The special structure of the developed ANN is shown on Fig. 5.

The main modules of the proposed ANN structure are convolutional layers. To avoid the network overfitting problem to the ANN it is added the layers of random transmission shutoffs of the particular outputs (dropout) with a probability of outputs disconnection equal to $25 \%$.

The last two layers of ANN are dense (fully connected) layers. A formed structure of the ANN outputs based on the requirements of the solved problem is following: an array of 200 outputs for each segment (P, QRS, T), i.e. total of 600 outputs.

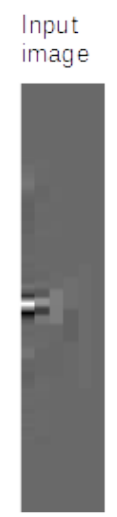

$6 \times 200 \times 1$

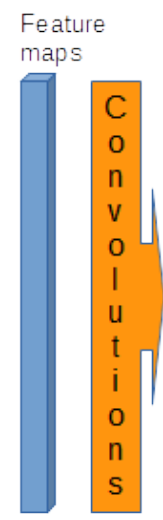

$6 \times 200 \times 32$

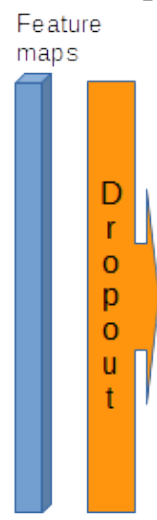

$6 \times 200 \times 32$

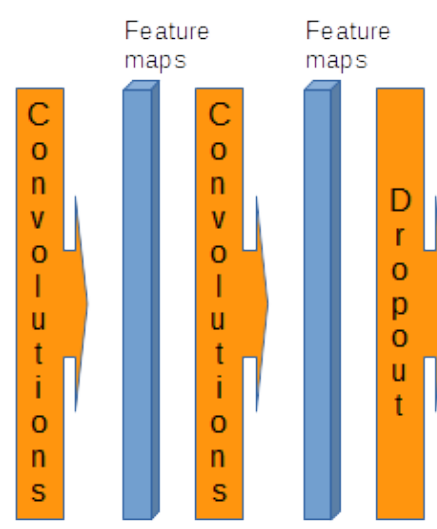

$6 \times 200 \times 64$

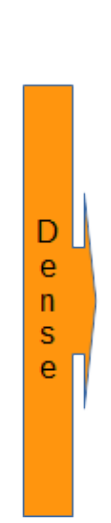

$6 \times 200 \times 64$

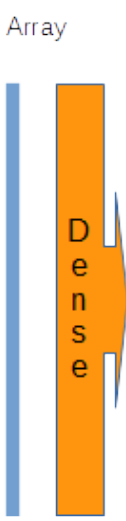

200

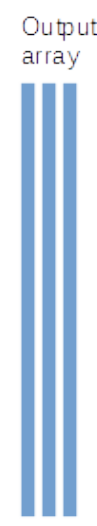

600

Figure 5. The structure of proposed convolutional artificial neural network.

\section{Experiments}

To train the recognition system, it is necessary to form a correct training sample base. Each training example should include the following: the image obtained as a result of the Haar wavelet transform of the average cardiocycle, as well as the coordinates of the start and end indexes of the segments (P, QRS, T) which was marked by the expert.

Before training the ANN, it was necessary to modify the training sample to bring the format of target responses for each training example in accordance with the ANN outputs. On the basis of a set of 6 coordinates of 3 segments from each cardiac cycle allocated by the cardiologist 3 array of outputs target values for each segment separately are formed according to the following formula (2):

$$
t^{m_{i}}=\left\{\begin{array}{l}
1, i \in\left[i^{m_{0}}, i^{m_{1}}\right] \\
0, i \notin\left[i^{m_{0}}, i^{m_{1}}\right]
\end{array}\right.
$$

where $\mathrm{i}=\{1 \ldots 200\}$ is the ANN's output index and the coordinate on the average cardiac cycle; $\mathrm{t}_{\mathrm{i}}^{\mathrm{m}}$ is the target output value; $\mathrm{m}$ is the segment number; $\mathrm{i}^{\mathrm{m}}{ }_{0}$ and $\mathrm{i}^{\mathrm{m}}{ }_{1}$ is the indexes of the start and end of the segment marked by the expert. 
After obtaining a set of outputs for each segment by using ANN it is necessary to interpret them. Each training example after transformation by formula (2) contain an image of the wavelet transform result of the average cardiocycle and an array of 600 values, the localization of each segment in which will be allocated units (Fig. 7).

The mean square error (MSE) is chosen as the optimization criterion in this work. The ANN's output MSE is the difference between the desired result (target) and the actual output. In the process of ANN fitting the regression problem is solved, i.e. the values of all outputs obtained during the work of ANN should match with all target outputs values in the training sample. To measure the recognition quality we use the standard error function:

$$
\text { MSE }=\frac{1}{m \cdot i} \sum_{m} \sum_{i}\left(e^{m_{i}}\right)^{2}=\frac{1}{m \cdot i} \sum_{m} \sum_{i}\left(t^{m_{i}}-y^{m_{i}}\right)^{2},
$$

where $e^{m}{ }_{i}$ is the error value of the segment's $m$ output $i ; y_{i}$ is the output obtained by ANN (depends on the ANN's current weights values and the input example); $\mathrm{t}_{\mathrm{i}}^{\mathrm{m}}$ is the target output value.

The ANN learning process is reduced to correcting the ANN weights, so as to minimize the MSE. The gradient descent method is generally used to minimize the MSE. In this work, an adaptive Adam algorithm based on gradient descent with smoothed versions of the mean and standard gradients is used to optimize MSE. [17]

Training samples base consisting of 1500 elements was used to fit the ANN. All samples were divided into training and test subsamples with the ratio of $80 \%$ by $20 \%$, i.e. training subsample contains 1200 examples and the test subsample contains 300 examples.

The average period of the ANN's training was 1 hour and 40 minutes on Lenovo G505s laptop (CPU AMD A10-5750M, RAM 4 GB). The graph of the error function obtained during ANN training is shown on Fig. 6, where the abscissa axis is the learning epoch number.

Training error displays the ANN's fitting accuracy on a training subsample. However, it does not give a full confidence that the trained model will also be working well on a new data that was not in the training subsample. The model accuracy on the new data is the ANN generalizing ability. To estimate the model generalization ability, the generalization error obtained on the test subsample was used (Fig. 6).

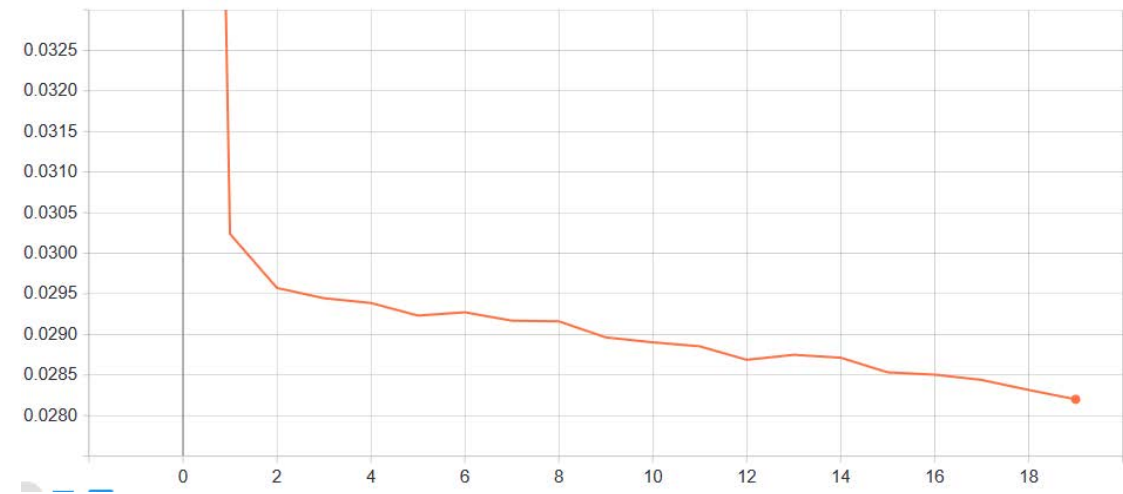

a) the training subsample error

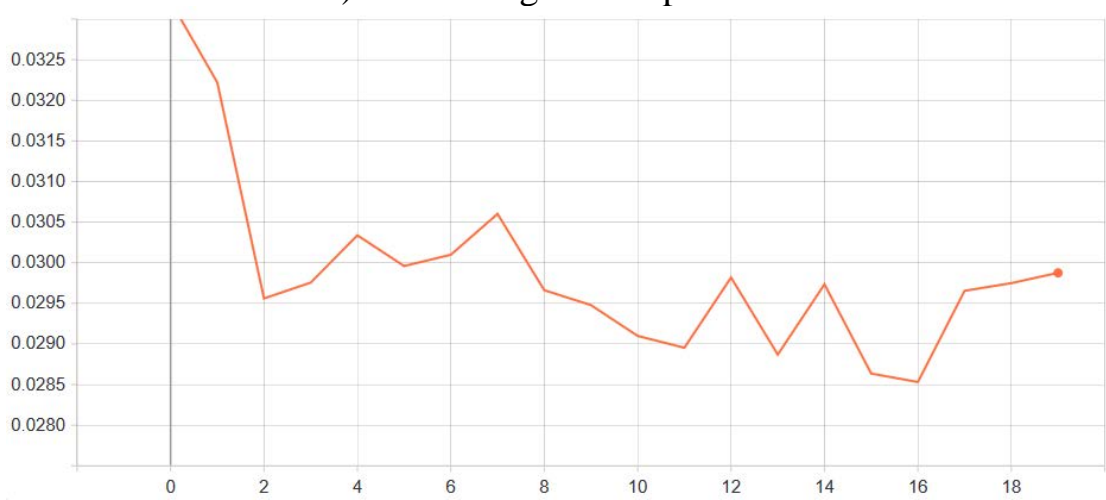

b) the generalization error

Figure 6. Changing an errors when training ANN. 
The ANN learning process is iterative. Each iteration is called an epoch. During one epoch, all the examples from the training subsample are submitted to the ANN input. It is also possible to validate the fitting error on the test subsample. As can be seen on the charts, the error function reaches a minimum at epoch 19.

After the ANN training phase, it is possible to use the cardiac cycle segments recognition system on unknown signals. During operation, as well as during training, the wavelet Haar transform of the average cardiocycle is applied, and then the resulting image is fed to the ANN input. The ANN was localized precisely enough the $\mathrm{P}$ and QRS segments on the EGG signal which neither was in the training nor in the test subsample (Fig. 7). Segment $T$ localization error could be the result of an inaccurate marking by the expert.
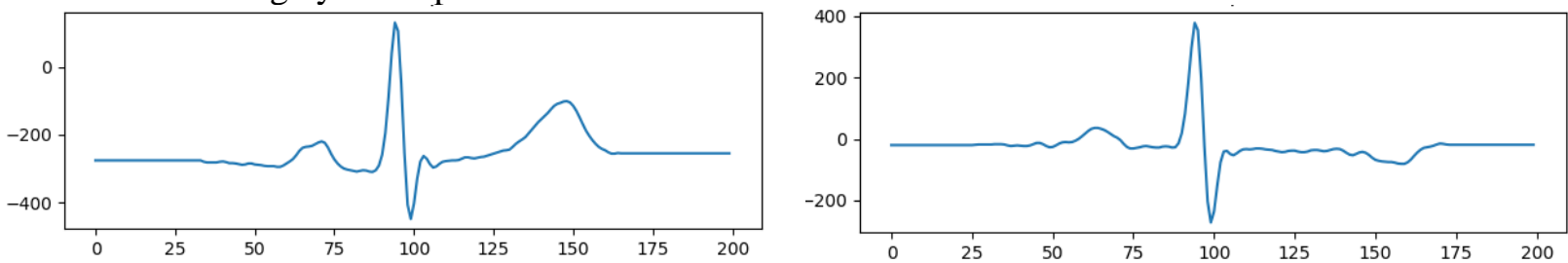

a) the average cardiac cycle signal
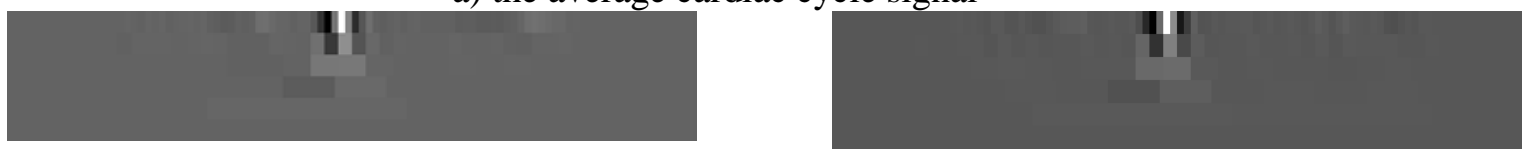

b) the Haar wavelet transform result (ANN input)
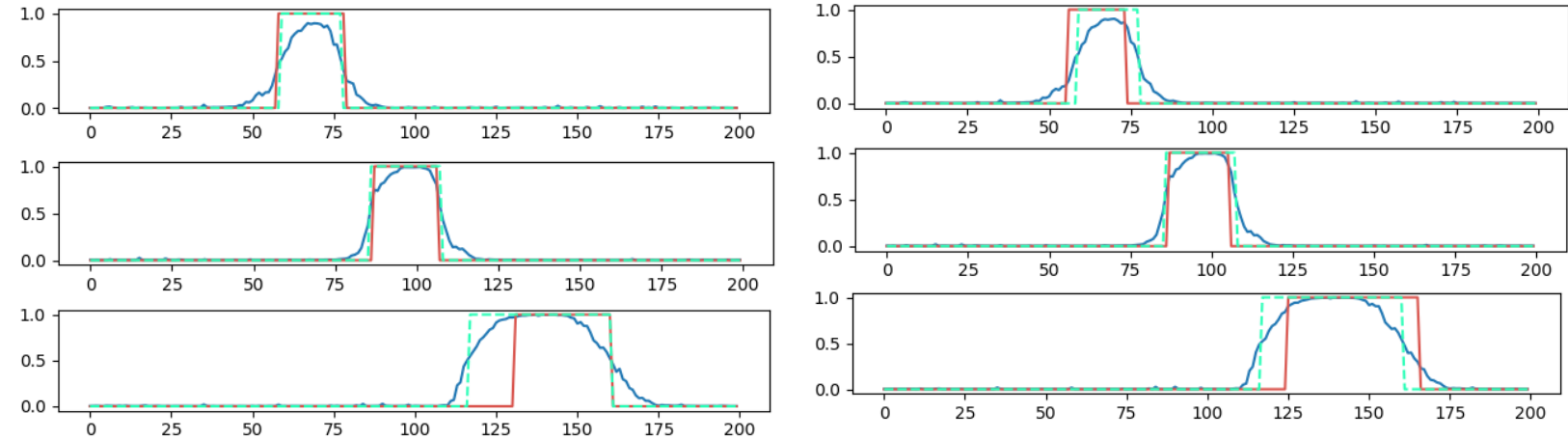

c) the segments recognition result (ANN outputs)

Figure 7. The example of segments localization results.

To perform experiments with the ECG morphology analysis system based on the proposed ANN, the Keras library was used - an open-source ANN library written in Python. It is capable of running on top of the Deeplearning4j, TensorFlow, or Theano frameworks. In this work, Keras was used along with TensorFlow - an open-source software library for machine learning. [18-19]

The Table 1 is showing the results of practical comparison of the proposed cardiocycle segments recognition system (based on convolutional ANN) and the previous version of recognition system proposed in [2] (pattern recognition algorithm) both carried out on the same signals sample (1500 samples). Column names mean the following:

a) $e_{\text {limit }}$ is the absolute difference between manual marking and automatic recognition of a segment coordinate, the error expressed in counts;

b) point is the name of the cardiac cycle characteristic points: $P_{0}, P_{1}, Q, S, T_{0}, T_{1}$;

c) \% is percentage of samples satisfying the value $e_{\text {limit }}$.

According to the clinical requirements for the quality of the algorithms results for the cardiocycles segments localization on real signals, a maximum error of no more than $40 \mathrm{MS}$ is allowed. In our case, when the quantization frequency is of $200 \mathrm{~Hz}$, maximum allowable error is equal 8 counts.

According to these requirements, the recognition system based on ANN with a maximum allowable error of 8 counts showed satisfactory results for the following segments (rows in table 1 marked are grayed out): $\mathrm{P}-82.18 \%$ of samples, QRS $-88.74 \%$ of samples, and $\mathrm{T}-85.36 \%$ of samples. The 
system based on the pattern recognition proposed in [2] showed a satisfactory results for the following segments: P $-81.44 \%$ of samples, QRS $-89.20 \%$ of samples, and T $-80.50 \%$ of samples.

Table 1. The results of the recognition systems comparison.

\begin{tabular}{cccc}
\hline & & \multicolumn{2}{c}{$\%$} \\
\cline { 3 - 4 } $\mathrm{e}_{\text {limit }}$ & point & $\begin{array}{c}\text { pattern } \\
\text { recognition [2] }\end{array}$ & $\begin{array}{c}\text { convolutional } \\
\text { ANN }\end{array}$ \\
\hline 2 & $P_{0}$ & 39.77 & 34.15 \\
\hline 2 & $P_{1}$ & 41.59 & 39.31 \\
\hline 2 & $Q$ & 58.76 & 46.25 \\
\hline 2 & $S$ & 60.64 & 57.18 \\
\hline 2 & $T_{0}$ & 43.46 & 45.69 \\
\hline 2 & $T_{1}$ & 43.62 & 41.27 \\
\hline 4 & $P_{0}$ & 58.33 & 53.28 \\
\hline 4 & $P_{1}$ & 60.89 & 56.34 \\
\hline 4 & $Q$ & 76.76 & 73.56 \\
\hline 4 & $S$ & 77.93 & 68.93 \\
\hline 4 & $T_{0}$ & 64.58 & 62.80 \\
\hline 4 & $T_{1}$ & 61.58 & 60.56 \\
\hline 8 & $P_{0}$ & 81.44 & 84.93 \\
\hline 8 & $P_{1}$ & 83.35 & 82.18 \\
\hline 8 & $Q$ & 90.38 & 90.82 \\
\hline 8 & $S$ & 89.20 & 88.74 \\
\hline 8 & $T_{0}$ & 84.65 & 86.15 \\
\hline 8 & $T_{1}$ & 80.50 & 85.36 \\
\hline 16 & $P_{0}$ & 93.54 & 96.93 \\
\hline 16 & $P_{1}$ & 93.95 & 92.99 \\
\hline 16 & $Q$ & 95.31 & 94.53 \\
\hline 16 & $S$ & 95.31 & 95.18 \\
\hline 16 & $T_{0}$ & 94.56 & 93.62 \\
\hline 16 & $T_{1}$ & 94.15 & 92.23 \\
\hline & & &
\end{tabular}

\section{Conclusion}

The system based on convolutional ANN proposed in this paper showed results slightly superior to the previous system based on pattern recognition [2] and similar to the work [20].

The proposed system effectively solves the problem used the standard modules of modern convolutional ANN, which simplifies the development of signal and image analysis systems.

The obtained results should be considered as satisfactory given that the training sample was formed mainly on the basis of the ECG from the functional diagnosis cardiological department with large percentage of it were pathological ECG [3].

In the future researches it is possible to modify the developed ANN structure by adding new layers to ANN. It is also necessary to increase the variety and volume of training sample signals. Thus, it is possible to improve the recognition system and use it in a clinical practice.

\section{References}

[1] Velic M, Padavic I. and Car S 2013 Computer aided ECG analysis - State of the art and upcoming challenges IEEE EUROCON International conference on computer as a tool DOI:10.1109/EUROCON.2013.6625218

[2] Korobeynikov A V 2004 Algorithms and software of computer monitoring systems for analysis of electrocardiograms morphology and rhythm (Doctoral dissertation, ISTU, Izhevsk, Russia)

[3] Kalyadin N I, Lemenkov V A, Korobeinikov A V, Perevozchikov S M, Odiyankov E G, Rodionov A N and Kotkov S M 2002 Development and Clinical Experience with a Computer 
Monitoring System Used at the Intensive Cardiological Care Unit. Biomedical Engineering 36(1) 44-49 DOI: 10.1023/a:1015417424098

[4] IzhTeleMed Ltd. (n.d.). URL: http://izhtelemed.ru/

[5] Li W 2019 Wavelets for Electrocardiogram: Overview and Taxonomy IEEE Access 7 2562725649 DOI: 10.1109/access.2018.2877793

[6] Lannoy G D, Frenay B, Verleysen M and Delbeke J 2009 Supervised ECG Delineation Using the Wavelet Transform and Hidden Markov Models IFMBE Proceedings 4th European Conference of the International Federation for Medical and Biological Engineering 22-25 DOI: 10.1007/978-3-540-89208-3_7

[7] Zaniol C, Varriale M C and Manica E 2018 Apnea Recognition with Wavelet Neural Networks TEMA (São Carlos) 19(2) 277 DOI: 10.5540/tema.2018.019.02.277

[8] Mallat S G, Peyré G 2009 A wavelet tour of signal processing: The sparse way (Amsterdam: Academic Press)

[9] LeCun A and Bengio Y 1995 Convolutional Networks for Images, Speech, and Time-Series, in Arbib (The Handbook of Brain Theory and Neural Networks, MIT Press)

[10] Özbay Y, Ceylan R and Karlik B 2006 A fuzzy clustering neural network architecture for classification of ECG arrhythmias Computers in Biology and Medicine 36(4) 376-388 DOI: 10.1016/j.compbiomed.2005.01.006

[11] Ronneberger O, Fischer P and Brox T 2015 U-Net: Convolutional Networks for Biomedical Image Segmentation Lecture Notes in Computer Science Medical Image Computing and Computer-Assisted Intervention 234-241 DOI: 10.1007/978-3-319-24574-4_28

[12] Hinton G, Deng L, Yu D, Dahl G, Mohamed A, Jaitly N and Kingsbury B 2012 Deep Neural Networks for Acoustic Modeling in Speech Recognition: The Shared Views of Four Research Groups IEEE Signal Processing Magazine 29(6) 82-97 DOI: 10.1109/msp.2012.2205597

[13] Deng L, Hinton G and Kingsbury B 2013 New types of deep neural network learning for speech recognition and related applications: An overview IEEE International Conference on Acoustics, Speech and Signal Processing DOI: 10.1109/icassp.2013.6639344

[14] Efitorov A and Dolenko S 2018 A New Type of a Wavelet Neural Network Optical Memory and Neural Networks 27(3) 152-160 DOI: 10.3103/s1060992x18030050

[15] Shoaib M, Shamseldin A Y, Melville B W and Khan M M 2016 Hybrid Wavelet Neural Network Approach Artificial Neural Network Modelling. Studies in Computational Intelligence 628

[16] Wasserman P D 1989 Neural computing: Theory and practice (New York, NY: Van Nostrand Reinhold)

[17] Goodfellow I, Bengio Y and Courville A 2017 Deep Learning (MIT Press) URL: http://www.deeplearningbook.org

[18] TensorFlow (n.d.) URL: https://www.tensorflow.org/

[19] Pattanayak S 2017 Introduction to Deep-Learning Concepts and TensorFlow Pro Deep Learning with TensorFlow 89-152 DOI: 10.1007/978-1-4842-3096-1_2

[20] Sampath A and Sumithira T 2016 ECG Morphological Marking using Discrete Wavelet Transform Intelligent Decision Technologies 10(4) 373-383 DOI: 10.3233/idt-160264 